\title{
In search of an optimal kernel for a bias correction method for density estimators
}

\author{
Lyudmila Sakhanenko * \\ Michigan State University
}

September 25, 2016

\begin{abstract}
We consider a bias correction method for kernel density estimators based on a generalized jack-knifing with different bandwidths. We compare it with a standard kernel density estimation method with fourth order kernels, since both methods have the same rate of convergence. The bias corrected method has a tuning parameter. We investigate how to optimize the constant in the asymptotical mean integrated squared error of the bias corrected estimator with respect to the tuning parameter. We also explore whether an optimal kernel exists. This paper answers on the questions posed in section 3.2 of [3] where only numerical investigation was given.

Key words: kernel smoothing, integrated mean square error, density estimation
\end{abstract}

\section{Introduction}

We consider the classical problem of nonparametric estimation of a density function $f(x), x \in$ $\mathbb{R}$, based on a simple random sample $X_{1}, \ldots, X_{n}$. A well-known kernel density estimator is $\hat{f}_{n, h_{n}}(x)=\frac{1}{n h_{n}} \sum_{i=1}^{n} K\left(\frac{x-X_{i}}{h_{n}}\right), x \in \mathbb{R}$, with a kernel $K$ and a bandwidth sequence $h_{n}$. It is a well studied and a popular estimator. Under certain conditions it enjoys many nice properties. In particular, for a 2-times differentiable density $f$ and a symmetric probability density kernel $K$ with a finite second moment, the asymptotical mean integrated squared error (AMISE) of the kernel density estimator is of the order $n^{-4 / 5}$, see the books [1] and [9] and references therein. Given an arbitrary positive number $r \neq 1$ construct an estimator of the form

$$
\tilde{f}_{n, r}(x)=\frac{r^{2}}{r^{2}-1} \hat{f}_{n, h_{n}}(x)-\frac{1}{r^{2}-1} \hat{f}_{n, r h_{n}}(x), x \in \mathbb{R} .
$$

*Department of Statistics and Probability, Michigan State University, East Lansing, MI 48824, luda@stt.msu.edu. Research is partially supported by NSF grant DMS-1208238. 
This is example 2.1 in [3]. It is called a generalized jack-knifing with different bandwidths in [3]. Under additional moment condition $\mu_{4, K}=\int w^{4} K(w) d w<\infty$ the bias of $\tilde{f}_{n, r}$ is $\frac{-r^{2}}{24} h_{n}^{4} f^{I V}(x) \mu_{4, K}\left(1+O\left(h_{n}\right)\right)$. Then the corresponding AMISE is

$$
A M I S E(\tilde{f}, h)=\frac{1}{n h}\left[\frac{r^{4}+r^{-1}}{\left(r^{2}-1\right)^{2}} R(K ; 1)-\frac{2 r}{\left(r^{2}-1\right)^{2}} R(K ; r)\right]+\frac{r^{4}}{(4 !)^{2}} h^{8} \mu_{4, K}^{2} R\left(f^{I V} ; 1\right),
$$

where $R(K ; r)=\int K(w) K\left(\frac{w}{r}\right) d w$. Then, with the optimal bandwidth the AMISE is minimized and is equal to

$$
A \tilde{M I S E}=n^{-8 / 9} \frac{9}{8}\left(\frac{1}{72}\right)^{1 / 9} \tilde{C}_{K}(r) R\left(f^{I V} ; 1\right)^{1 / 9}
$$

with

$$
\tilde{C}_{K}(r)=\mu_{4, K}^{2 / 9} r^{4 / 9}\left[\frac{r^{4}+r^{-1}}{\left(r^{2}-1\right)^{2}} R(K ; 1)-\frac{2 r}{\left(r^{2}-1\right)^{2}} R(K ; r)\right]^{8 / 9} .
$$

Note that the rate of convergence is now of the order $n^{-8 / 9}$, which corresponds to so-called fourth order kernels whose first 3 moments are 0 while the fourth moment is finite and nonzero. Indeed, the second order kernel $K$ is transformed into the fourth order kernel

$$
L_{r}(u)=\frac{r^{2}}{r^{2}-1} K(u)-\frac{1}{r^{2}-1} \frac{K(u / r)}{r}, \quad u \in \mathbb{R} .
$$

This is one of many approaches of bias reduction and improvement of the rate of convergence proposed in literature over the years; see e.g. [3, 4]. This problem keeps attracting researchers. For example, [5] offers yet another bias correction method. The approach considered in this paper has rekindled our interest after seeing its use in a recent work [7] on an integral curve estimation with an application to brain imaging.

The goal of this paper is to investigate how to choose an optimal $r$ and then how to choose $K$ to minimize AMISE if possible. The authors of [3] pointed out that the choice of $r$ depends heavily on the choice of $K$. They obtained only numerically some optimal $r$ values for 3 common kernel functions and claimed that the performance does not suffer if non-optimal $r$ is used. On contrary, we find significant effect and thus we investigate this problem further.

First, we choose a number of kernels and calculate the corresponding constants. Tables 1 and 2 summarize these preliminary findings. There $U$ stands for uniform density on $[-1$, 1] for the kernel; $N$ is the standard normal density; $K_{2}$ is famous Epanechnikov's kernel; $\Delta$ is a triangular kernel; $M$ is a mollifier with support $[-1,1]$. The rest of kernels are defined to put an infinite mass at 0 while they have the finite support. Indeed, $L$ blows up at 0 logarithmically, while kernels $P_{\alpha}, \alpha \in(0,0.5)$, blow up at 0 polynomially. Figure 1 shows typical densities as those used in [1], called corner functions there, together with their estimators $\tilde{f}_{n, r}$ given for kernels $N, U, P_{0.05}$.

We use the same notations as in [2], where one can find detailed formulas of MISE of some of these kernels. The second column in Table 2 contains the values of the kernel 

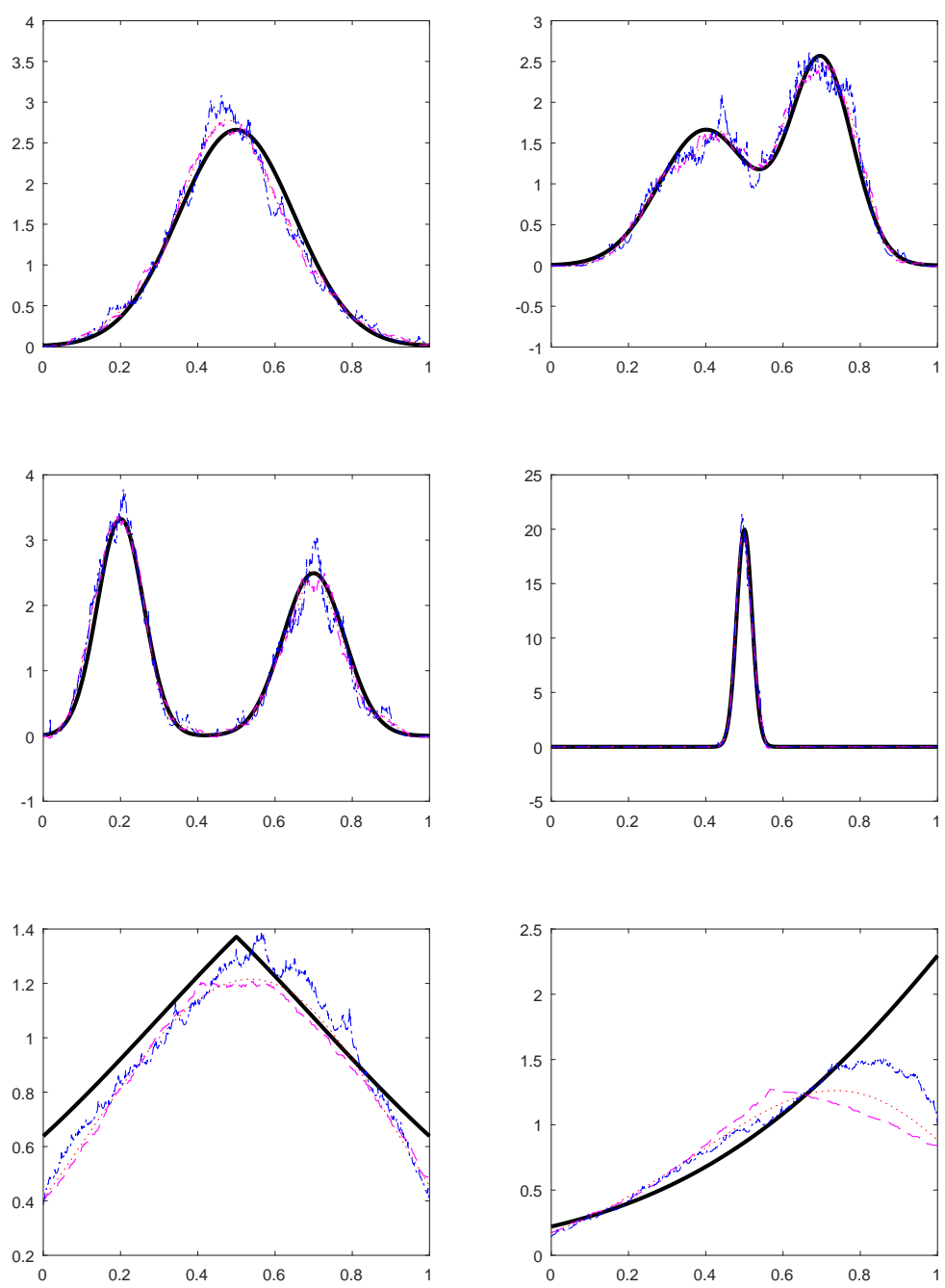

Figure 1: Kernel density estimators are given for normal, bimodal, strata, delta, angle, and monotone densities as defined on pp. 18-19 in [1]. The solid thick line correspond to the underlying density, while estimated densities are shown in red dotted, magenta dash-dotted, blue dashed for normal, uniform and $P_{0.05}$ kernels respectively. 


\begin{tabular}{|l|l|l|l|}
\hline Kernel & $K$ & $R(K ; 1)$ & $R(K ; r)$ \\
\hline$U$ & $0.5 I(|w| \leq 1)$ & 0.5 & $0.5(r \wedge 1)$ \\
\hline$N$ & $\frac{e^{-0.5 w^{2}}}{\sqrt{2 \pi}}$ & $\frac{1}{2 \sqrt{\pi}}$ & $\frac{r}{\sqrt{2 \pi\left(1+r^{2}\right)}}$ \\
\hline$K_{2}$ & $0.75\left(1-w^{2}\right) I$ & 0.6 & $\frac{3}{4}\left(r-\frac{r^{3}}{5}\right) I(r<1)+\frac{3}{4}\left(1-\frac{1}{5 r^{2}}\right) I(r>1)$ \\
\hline$\Delta$ & $(1+|w|) I(|w| \leq 1)$ & $\frac{2}{3}$ & $\left(r-\frac{r^{2}}{3}\right) I(0<r<1)+\left(1-\frac{1}{3 r}\right) I(r>1)$ \\
\hline$M$ & $2.2523 \exp \left\{-\frac{1}{1-w^{2}}\right\}$ & 0.6747 & $10.1453 \int_{0}^{r \wedge 1} \exp \left\{-\frac{1}{1-x^{2}}-\frac{r^{2}}{r^{2}-x^{2}}\right\} d x$ \\
\hline$L$ & $-0.5 \ln |w| I(|w| \leq 1)$ & 1 & {$\left[r(\ln r)^{2}-\frac{3}{2} \ln r+r\right] I(r<1)+\left[1+\frac{1}{2} \ln r\right] I(r>1)$} \\
\hline$P_{\alpha}$ & $\frac{1-\alpha}{2 \alpha}\left(|w|^{-\alpha}-1\right) I(|w| \leq 1)$ & $\frac{1-\alpha}{1-2 \alpha}$ & $\frac{1-\alpha}{2 \alpha}\left[\left(\frac{r^{1-\alpha}}{1-2 \alpha}-r\right) I(r<1)+\left(\frac{r^{\alpha}}{1-2 \alpha}-1\right) I(r>1)\right]$ \\
\hline
\end{tabular}

Table 1: Various kernel functions $K$ and the associated $R(K ; \cdot)$.

\begin{tabular}{|l|l|l|l|l|}
\hline Kernel & $\tilde{C}_{K}\left(r_{0}\right)$ & $r_{0}$ & $g_{K}\left(r_{0}\right)$ & $\mu_{4, K}$ \\
\hline$U$ & 0.6866 & $0.4646 ; 2.152$ & 0.9795 & 0.2 \\
\hline$N$ & 0.6599 & 1 & 0.476 & 3 \\
\hline$K_{2}$ & 0.6241 & $0.671 ; 1.491$ & 1.0875 & $\frac{3}{35}$ \\
\hline$\Delta$ & 0.6283 & $0.806 ; 1.2407$ & 6.6497 & $\frac{1}{15}$ \\
\hline$M$ & 0.6501 & $0.421 ; 2.3753$ & 1.284 & 0.053 \\
\hline$L$ & 0.753 & 1 & 1.625 & 0.04 \\
\hline$P_{0.45}$ & 0.4123 & $0.3564 ; 2.8058$ & 0.936 & 0.0242 \\
\hline$P_{0.25}$ & 0.261 & $0.3548 ; 2.8185$ & 0.5234 & 0.0316 \\
\hline$P_{0.05}$ & 0.0655 & $0.3552 ; 2.8153$ & 0.1053 & 0.0384 \\
\hline
\end{tabular}

Table 2: Constants in AMISE for various kernel functions.

dependent constant in the optimal AMISE of the bias corrected estimator $\tilde{f}$. Denote

$$
g_{K}(r)=\sqrt{r}\left[\frac{r^{4}+r^{-1}}{\left(r^{2}-1\right)^{2}} R(K ; 1)-\frac{2 r}{\left(r^{2}-1\right)^{2}} R(K ; r)\right], r>0, r \neq 1 .
$$

The third column provides the points of minimum for $g_{K}$. The fourth column provides the minimum of $g_{K}$ over $r \in(0, \infty)$. Note that all kernels are of the second order, so the classical kernel density estimator achieves the slower rate of convergence $n^{-4 / 5}$ and it has a different AMISE; see e.g. [9].

Naturally, the bias corrected estimator has an additional advantage of having a tuning parameter $r$. Using the best $r$ for the particular kernel can significantly improve AMISE. An immediate question arises to find a simple closed-form solution to the minimization problem $\min _{0<r<\infty} g_{K}(r)$. Figure 2 demonstrates the difficulty of searching for the optimal $r$ empirically and numerically. In this paper we search for the optimal $r$ theoretically.

We give a partial answer to the above question. In particular, for kernels supported on the whole real line $\mathbb{R}$ under some restrictions we have analytically obtained the minimum with respect to $r$ of AMISE for $\tilde{f}$. Then we have posed a follow-up question of finding the optimal kernel in this class that minimizes AMISE with respect to $K$. We have proved that no such kernel exists. Intuitively, it is quite natural since fourth order kernels could 


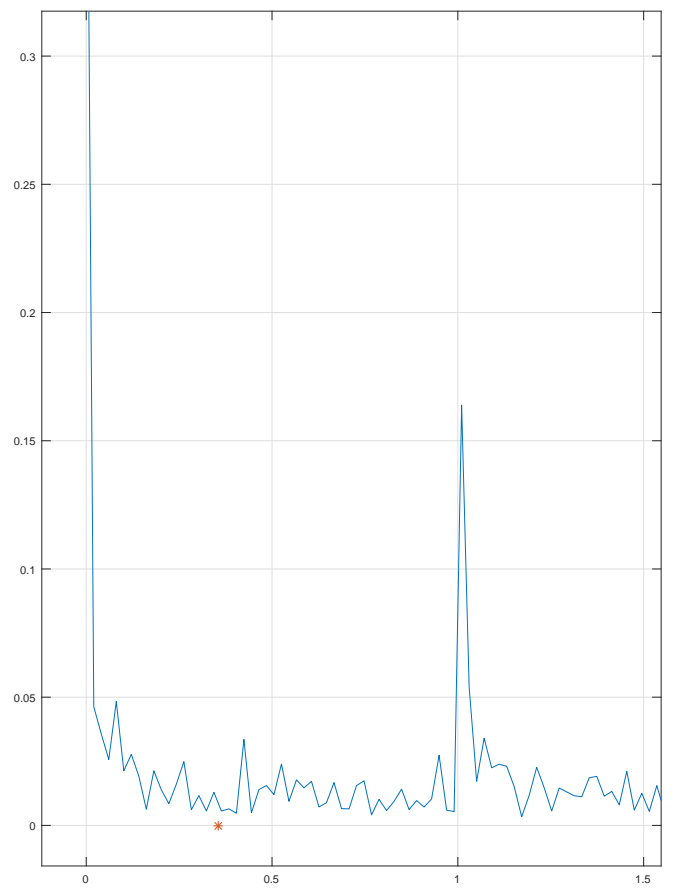

Figure 2: Empirical MSE as a function of $r$ for kernel $P_{0.05}$ and the normal underlying density. The optimal $r$ is shown by a star.

be further sped up by a similar principle to produce sixth order kernels with an even better convergence rate.

For kernels with bounded support the questions of finding an optimal $r$ and then finding of an optimal kernel remain open. However, as one can see from Table 2 that kernels with bounded support can have very small constants $\tilde{C}_{K}\left(r_{0}\right)$ if they are allowed to be unbounded, see the example of kernel $P_{0.05}$. Also by looking at Table 2 one could wonder if for bounded kernels the constant does not vary too much. We have investigated this question as well.

Overall, the bias corrected estimator enjoys the similar asymptotical properties as the classical kernel density estimator. Both are asymptotically normal at each fixed $x$.

Finally, we mention that the estimator $\tilde{f}$ with a particular choice of $r=2$ is a special example of the kernel $M_{2}$ in a recent work on bias reduction [6]. We are interested in optimizing over $r$, while in [6] a general approach is developed for kernel estimators with higher order kernels.

The rest of the paper is organized as follows. We introduce notation and main results in section 2, followed by conclusion and discussion in section 3. Proofs are presented in section 4 . 


\section{Main results}

Note that $g_{K}$ is nonnegative everywhere due to its definition through variance. The following proposition describes the basic properties of $g_{K}$ which are dependent on the behavior of the kernel $K$.

Proposition 1 (a) For any symmetric kernel $K$ the function $g_{K}$ is invariant under inversion, i.e. $g_{K}\left(r^{-1}\right)=g_{K}(r)$ for all $r>0$.

(b) Let any symmetric continuously differentiable everywhere kernel $K$ have a connected support in $\mathbb{R}$. Then for such a kernel there exists the limit

$$
\lim _{r \rightarrow 1} g_{K}(r)=\frac{3}{2} R(K ; 1)+\frac{1}{4} \int w^{2}\left(K^{\prime}(w)\right)^{2} d w=: g_{K}(1)
$$

so the function $g_{K}$ is continuous at 1 .

We remark that condition (b) is violated if the support of $K$ consists of several disjoint intervals. Due to symmetry the support is $(-M, M)$ with a finite or infinite $M>0$.

Theorem 1 For any continuously differentiable symmetric kernel $K$ satisfying conditions in Proposition 1 (b) with infinite support $(-\infty, \infty)$ such that $\int K\left(\frac{w}{r}\right)\left[K(w)+w K^{\prime}(w)\right] d w>$ 0 for all $r \in(1,3)$, the minimum of $g_{K}$ over $(0, \infty)$ is attained only at $r_{0}=1$. In this case $L_{1}(u)=0.5 u K^{\prime}(u)+1.5 K(u), u \in \mathbb{R}$.

Theorem 2 Let $K$ be a kernel satisfying conditions in Proposition 1 (b) such that it is monotone on $\mathbb{R}_{+}$and $\int K(w) K\left(\frac{w}{r}\right) d w>0$ for all $r \in(0,1)$. Then the minimum of $g_{K}$ over $(0, \infty)$ is attained at a point or points $r_{0}$ in the interval $(0.3382,1]$ and at a point or points $r_{0}^{-1}$.

Note that Theorem 1 is a special partial case of Theorem 2. The constant 0.3382 is an approximation of the exact root of the following function $-r^{6}+11 r^{4}-2 r^{2}+9 r-r^{-1}$ on the interval $r \in[0,1]$. The standard normal kernel satisfies Theorem 1 . We remark that one can allow continuous differentiability everywhere except at a finite number of points.

It is natural to ask whether one can choose an optimal kernel that would minimize the constant $\tilde{C}_{K}\left(r_{0}\right)$ in the AMISE. We can answer on this question for the kernels in Theorem 1. Note that under conditions of Theorem 1 we have $r_{0}=1$ and $\tilde{C}_{K}(1)=\left(g_{K}(1) \mu_{4, K}^{1 / 4}\right)^{8 / 9}$. Thus, consider the following minimization problem

$$
\begin{array}{ll}
\min _{K \in \mathcal{K}} & g_{K}(1) \mu_{4, K}^{1 / 4}= \\
\min _{K \in \mathcal{K}} & {\left[1.5 \int K^{2}(w) d w+0.25 \int\left(w K^{\prime}(w)\right)^{2} d w\right]\left|\int w^{4} K(w) d w\right|^{1 / 4},}
\end{array}
$$

where the class $\mathcal{K}$ consists of kernels satisfying the conditions of Theorem 1 . It is quite obvious that the minimization problem (1) has solutions that are only kernels of fourth 
order, which we need to exclude since we are comparing density smoothing methods that yield the order $n^{-8 / 9}$. As a result, one should consider the minimization problem

$$
\begin{array}{ll}
\min _{K \in \mathcal{K}} & g_{K}(1)= \\
\min _{K \in \mathcal{K}} & {\left[1.5 \int K^{2}(w) d w+0.25 \int\left(w K^{\prime}(w)\right)^{2} d w\right] .}
\end{array}
$$

Unfortunately, this problem does not have a solution in the class of kernels $\mathcal{K}$ as summarized in the theorem below. No solution exists in $\mathcal{K}$ even if a constraint such as $\int w^{2} K(w) d w=1$ added.

Theorem 3 Any kernel in $\mathcal{K}$ with zero fourth moment $\mu_{4, K}=\int w^{4} K(w) d w=0$ minimizes (1). The minimization problem (2) does not have a solution.

However, one can see that problem (2) has a trivial solution $K=0$ a.e. in the class of functions in $\mathcal{L}^{2}(\mathbb{R})$ that are differentiable almost everywhere such that $w K^{\prime}(w)$ is also square integrable on $\mathbb{R}$. Technically, this minimizer can be approximated by sequences of kernels in $\mathcal{K}$.

Recall that $R(K ; 1)=\int K^{2}(w) d w$. Denote $J(K)=\int w^{2}\left(K^{\prime}(w)\right)^{2} d w$. Let $D(K)=$ $\min _{r \in(0.3382,1)}(-1) \int w^{3} K^{\prime \prime}(w) K^{\prime}(w / r) d w$.

Theorem 4 For any kernel satisfying the conditions of Theorem 2 one of the following 3 cases holds. then

(a) If $D(K)<-18.3973 R(K ; 1)+2.9496 J(K)$ and $D(K)<-0.75 R(K ; 1)+1.5 J(K)$

$$
\min _{r \in(0,+\infty)} g_{K}(r) \geq 1.8665 R(K ; 1)+0.1098 J(K)+0.0716 D(K) .
$$
then

(b) If $D(K)>-18.3973 R(K ; 1)+2.9496 J(K)$ and $D(K)<-0.75 R(K ; 1)+1.5 J(K)$

$$
\min _{r \in(0,+\infty)} g_{K}(r) \geq 0.5486 R(K ; 1)-0.3174 J(K) .
$$
then

(c) If $D(K)>-18.3973 R(K ; 1)+2.9496 J(K)$ and $D(K)>-0.75 R(K ; 1)+1.5 J(K)$

$$
\min _{r \in(0,+\infty)} g_{K}(r)=g_{K}(1)=1.5 R(K ; 1)+0.25 J(K) .
$$

We remark that $\min _{r \in(0,+\infty)} g_{K}(r) \leq 1.5 R(K ; 1)+0.25 J(K)$. And the difference between lower bounds in Theorem 4 and this upper bound is negligible in comparison with the value of the minimum. Instead of solving the exact minimization problem in Theorem 3 one can solve an approximate minimization problem of the lower bounds in Theorem 4 (a) or (b).

Naturally, consider the minimization problem

$$
\min _{K \in \mathcal{K}_{b}}\left[0.5486 \int K^{2}(w) d w-0.3174 \int\left(w K^{\prime}(w)\right)^{2} d w\right]
$$


where kernels from $\mathcal{K}_{b}$ satisfy conditions of Theorem 2 and condition of Theorem 3 (b). Using the same arguments as in the proof of Theorem 3 , we can show that there is no solution inside $\mathcal{K}_{b}$. On the boundary of $\mathcal{K}_{b}$ the minimization problem reduces to maximizing of $R(K ; 1)$ provided that $J(K)=12.17391 R(K ; 1)$ and $D(K)=17.511 R(K ; 1)$. This explains the success of kernels $P_{\alpha}$ in Table 2 .

Next, one would need to consider the minimization problem

$$
\min _{K \in \mathcal{K}_{a}}\left[1.8665 \int K^{2}(w) d w+0.1098 \int\left(w K^{\prime}(w)\right)^{2} d w-\max _{r \in(0.3382,1)} \int w^{3} K^{\prime \prime}(w) K^{\prime}(w / r) d w\right]
$$

where kernels from $\mathcal{K}_{a}$ satisfy conditions of Theorem 2 and condition of Theorem 3 (a). This is left for future investigation.

\section{Conclusion and discussion}

We consider a particular generalized jack-knifing method that corrects for bias and speeds up the convergence rate. The bias corrected method studied in this paper can yield estimators with reasonable savings in the AMISE compared to the standard kernel smoothing method based on fourth order kernels. It also has a tuning parameter. A natural question arises: how to choose an optimal tuning parameter?

We have showed that for a large subclass of symmetric kernels supported on the whole real line the optimal tuning parameter is 1 . This class includes the standard normal kernel. For symmetric kernels with bounded support we showed that optimal parameter values are in the interval $(0.3382,1]$, which is a modest improvement over the interval $(0,1]$ given in literature.

Then a next natural question arises: how to choose an optimal kernel? We show that for a large subclass of symmetric kernels supported on the whole real line no optimal kernel exists. However, there exist unbounded kernels with bounded support that could make AMISE very small. Examples of such kernels are provided. They give better constants than commonly used kernels such as uniform, standard normal, Epanechnikov's, triangular.

The problem of choosing an optimal tuning parameter and an optimal kernel remains open for kernels with a bounded support for the bias corrected method. But we have showed that the optimal constant is bounded from both sides by linear combinations of 3 integrals related to kernels. And the difference between these 2 bounds is negligible for many common kernels, so using the optimal constant would not dramatically improve the value of AMISE for kernels with bounded support.

\section{Proofs}

\subsection{Proof of Proposition 1}

(a) Simple algebra and observation that $R\left(K ; r^{-1}\right)=r^{-1} R(K ; r)$ yield the statement. 
(b) First note that one can use l'Hôpital's rule in order to calculate $\lim _{r \rightarrow 1} g(r)$. Then the limit in question is equal to

$$
\begin{aligned}
& \lim _{r \rightarrow 1} \frac{\sqrt{r}}{(r+1)^{2}} \cdot \frac{\left(r^{4}+r^{-1}\right) R(K ; 1)-2 r R(K ; r)}{(r-1)^{2}} \\
& =\frac{1}{4} \lim _{r \rightarrow 1} \frac{\left(4 r^{3}-r^{-2}\right) R(K ; 1)-2 R(K ; r)-2 r R^{\prime}(K ; r)}{2(r-1)} .
\end{aligned}
$$

Note that

$$
\begin{aligned}
R^{\prime}(K ; r) & =\int K(w) K^{\prime}\left(\frac{w}{r}\right)\left(-\frac{w}{r^{2}}\right) d w=\int K(w)\left(-\frac{w}{r}\right) d K\left(\frac{w}{r}\right) \\
& =M(r \wedge 1)\left[K(-M(r \wedge 1)) K\left(-M\left(1 \wedge r^{-1}\right)\right)-K(M(r \wedge 1)) K\left(M\left(1 \wedge r^{-1}\right)\right)\right] \\
& +\frac{1}{r} R(K ; r)+\frac{1}{r} \int w K^{\prime}(w) K\left(\frac{w}{r}\right) d w .
\end{aligned}
$$

Under the assumptions on the kernel it is equal to $\frac{1}{r} R(K ; r)+\frac{1}{r} \int w K^{\prime}(w) K\left(\frac{w}{r}\right) d w$. Moreover, at $r=1$ we have $R^{\prime}(K ; 1)=\frac{1}{2} R(K ; 1)$, so that one can apply l'Hôpital's rule again. Then $\lim _{r \rightarrow 1} g(r)=\frac{1}{8}\left(14 R(K ; 1)-4 R^{\prime}(K ; 1)-2 R^{\prime \prime}(K ; 1)\right)$. Note that

$$
\begin{aligned}
R^{\prime \prime}(K ; r) & =-\frac{1}{r^{2}} R(K ; r)+\frac{1}{r} R^{\prime}(K ; r)-\frac{1}{r^{2}} \int w K^{\prime}(w) K\left(\frac{w}{r}\right) d w \\
& -\frac{1}{r^{3}} \int w^{2} K^{\prime}(w) K^{\prime}\left(\frac{w}{r}\right) d w=-\frac{1}{r^{3}} \int w^{2} K^{\prime}(w) K^{\prime}\left(\frac{w}{r}\right) d w
\end{aligned}
$$

So $R^{\prime \prime}(K ; 1)=-\int w^{2}\left(K^{\prime}(w)\right)^{2} d w$ and as a result

$$
\lim _{r \rightarrow 1} g(r)=\frac{3}{2} R(K ; 1)+\frac{1}{4} \int w^{2}\left(K^{\prime}(w)\right)^{2} d w .
$$

Note that the limit is infinite for non-symmetric kernels with a finite support $[-M, M]$ and non-zero $K^{2}(M) M$. The proof of part (b) is complete.

\subsection{Proof of Theorems 1 and 2}

In order to find the points of minimum of $g$ it is natural to find the zeros and singularities of its derivative $g^{\prime}$. Straightforward algebra yields

$$
g^{\prime}(r)=\frac{\left(r^{6}-9 r^{4}-9 r+r^{-1}\right) R(K ; 1)+\left(10 r^{3}+6 r\right) R(K ; r)-4 r^{2}\left(r^{2}-1\right) R^{\prime}(K ; r)}{\left(r^{2}-1\right)^{3} 2 \sqrt{r}} .
$$

So the potential points of minimum of $g$ are among 0,1 , and the roots of the equation

$$
\left(r^{6}-9 r^{4}-9 r+r^{-1}\right) R(K ; 1)+\left(10 r^{3}+6 r\right) R(K ; r)=4 r^{2}\left(r^{2}-1\right) R^{\prime}(K ; r) .
$$


Consider $r \in(0,1)$. Then for kernels that are monotone on the half-lines $\mathbb{R}_{+}$and $\mathbb{R}_{-}$, $R^{\prime}(K ; r)$ is decreasing everywhere on $\mathbb{R}_{+}$, so $R^{\prime}(K ; r)>R^{\prime}(K ; 1)=\frac{1}{2} R(K ; 1)$ for $0<r<1$. From (4) we have no roots for $r$ satisfying

$4 r^{2}\left(r^{2}-1\right) R^{\prime}(K ; r)<2 r^{2}\left(r^{2}-1\right) R(K ; 1)<\left(r^{6}-9 r^{4}-9 r+r^{-1}\right) R(K ; 1)+\left(10 r^{3}+6 r\right) R(K ; r)$

or equivalently

$$
\left(r^{6}-11 r^{4}+2 r^{2}-9 r+r^{-1}\right) R(K ; 1)+\left(10 r^{3}+6 r\right) R(K ; r)>0,
$$

which can be rewritten as

$$
\frac{R(K ; r)}{R(K ; 1)}>\frac{-r^{6}+11 r^{4}-2 r^{2}+9 r-r^{-1}}{10 r^{3}+6 r}
$$

Since

$$
\frac{-r^{6}+11 r^{4}-2 r^{2}+9 r-r^{-1}}{10 r^{3}+6 r}<0
$$

on $(0,0.3382)$ while $\frac{R(K ; r)}{R(K ; 1)}>0$ everywhere, there are no roots of $(4)$ on $(0,0.3382)$. The proof of Theorem 2 is complete by applying Proposition 1 (a).

Furthermore, consider the denominator of $g^{\prime}$ on the interval $(1,1 / 0.3382) \subset(1,3)$. Denote it as $\varphi(r)$. Then

$$
\begin{aligned}
\varphi(r) & =\left(r^{6}-9 r^{4}+10 r^{3}-3 r+r^{-1}\right) R(K ; 1)+\left(10 r^{3}+6 r\right)[R(K ; r)-R(K ; 1)] \\
& -4 r^{2}\left(r^{2}-1\right) R^{\prime}(K ; r)=\left(r^{6}-9 r^{4}+10 r^{3}-3 r+r^{-1}\right) 2 R^{\prime}(K ; 1) \\
& +\left(10 r^{3}+6 r\right)(r-1) \int_{0}^{1} R^{\prime}(K ; \lambda r+1-\lambda) d \lambda-4 r^{2}\left(r^{2}-1\right) R^{\prime}(K ; r) \\
& <\left(r^{6}-2 r^{4}+r^{2}-4.5 r+r^{-1}\right) R^{\prime}(K ; r)-4 r^{2}\left(r^{2}-1\right) R^{\prime}(K ; r) \\
& +\left(r^{6}-16 r^{4}+10 r^{3}-r^{2}+1.5 r+r^{-1}\right) \int_{0}^{1} R^{\prime}(K ; \lambda r+1-\lambda) d \lambda \\
& +\left(10 r^{3}+6 r\right)(r-1) \int_{0}^{1} R^{\prime}(K ; \lambda r+1-\lambda) d \lambda \\
& =\left(r^{6}-5 r^{4}+5 r^{2}-4.5 r+r^{-1}\right)\left[R^{\prime}(K ; r)+\int_{0}^{1} R^{\prime}(K ; \lambda r+1-\lambda) d \lambda\right]<0,
\end{aligned}
$$

where in line (5) we notice that $\left(r^{6}-9 r^{4}+10 r^{3}-3 r+r^{-1}\right)<0$ on $(1,3)$ and $R(K ; 1)>0$; in line (6) we use $R(K ; 1)=2 R^{\prime}(K ; 1)$ and $R^{\prime}(K ; 1) \geq R^{\prime}(K ; \lambda r+1-\lambda)$ for all $\lambda \in[0,1]$ due to $R^{\prime \prime}(K ; r)<0$ and both expressions in round brackets are negative on $(1,3)$; in line $(7)$ we notice that $\left(r^{6}-5 r^{4}+5 r^{2}-4.5 r+r^{-1}\right)<0$ and $R^{\prime}(K ; r)+\int_{0}^{1} R^{\prime}(K ; \lambda r+1-\lambda) d \lambda>0$ on $[1,3]$ if $R^{\prime}(K ; r)>0$ for $r \in[1,3]$.

As a result, there are no roots of $g_{K}^{\prime}$ on the interval $[1,3]$. By Proposition 1 (a) and Theorem 2 the only point of minimum of function $g_{K}$ is $r=1$, which had to be proven.

Finally, by l'Hôpital's rule we get the expression for $L_{1}$. This completes the proof of Theorem 1. 


\subsection{Proof of Theorem 3}

First use the symmetry and rewrite the functional needed to minimize as

$$
\Phi(K ; \lambda)=\left[3 \int_{0}^{\infty} K^{2}(w) d w+0.5 \int_{0}^{\infty}\left(w K^{\prime}(w)\right)^{2} d w\right]-\lambda\left(\int_{0}^{\infty} K(w) d w-0.5\right)
$$

where $\lambda$ is a Lagrange multiplier. The variational derivative of $\Phi$ at $K$ in the direction $\Delta$ is

$$
\begin{aligned}
& \lim _{\varepsilon \rightarrow 0} \frac{\Phi(K+\varepsilon \Delta ; \lambda)-\Phi(K ; \lambda)}{\varepsilon}=6 \int_{0}^{\infty} K(w) \Delta(w) d w-2 \int_{0}^{\infty} w K^{\prime}(w) \Delta(w) d w \\
- & \int_{0}^{\infty} w^{2} K^{\prime \prime}(w) \Delta(w) d w-\lambda \int_{0}^{\infty} \Delta(w) d w .
\end{aligned}
$$

We set it equal to 0 for all integrable functions $\Delta$. Then we obtain the following integraldifferential equation with a boundary condition

$$
6 K(z)-2 z K^{\prime}(z)-z^{2} K^{\prime \prime}(z)=\lambda, \int_{0}^{\infty} K(w) d w=0.5 .
$$

First, consider $\lambda=0$. We solve the homogeneous differential equation

$$
6 K(z)-2 z K^{\prime}(z)-z^{2} K^{\prime \prime}(z)=0
$$

by the change of variable $L(t)=K\left(e^{t}\right)$, then $6 L(t)-L^{\prime}(t)-L^{\prime \prime}(t)=0$ with the characteristic equation $\lambda^{2}+\lambda-6=0$. So the general solution is $L(t)=a_{+} e^{\lambda_{+} t}+a_{-} e^{\lambda_{-} t}$, where $\lambda_{ \pm}=$ $-0.5 \pm 2.5$. The partial solution of $6 L(t)-L^{\prime}(t)-L^{\prime \prime}(t)=\lambda$ is $L(t)=\frac{\lambda}{6}$. So we have $L(t)=\frac{\lambda}{6}+a_{+} e^{2 t}+a_{-} e^{-3 t}$ as the general solution. However, any function of this class maximizes the functional $\Phi$ and there are no kernels in the corresponding class of $K$. So a minimizer does not exist. This completes the proof of Theorem 3.

\subsection{Proof of Theorem 4}

For $r \in(0,1)$ we have the following Taylor expansion using relationships $R^{\prime}(K ; 1)=$ $0.5 R(K ; 1)$ and $R^{\prime \prime}(K ; 1)=-J(K)$

$$
\begin{aligned}
R(K ; 1)-R(K ; 1) & =\int_{0}^{1} R^{\prime}(K ; \lambda+(1-\lambda) r)(1-r) d \lambda \\
& =(1-r)\left[R^{\prime}(K ; 1)-\int_{0}^{1} \lambda R^{\prime \prime}(K ; \lambda+(1-\lambda) r)(1-r) d \lambda\right] \\
& =0.5(1-r) R(K ; 1)+0.5(1-r)^{2} J(K) \\
& +0.5(1-r)^{3} \int_{0}^{1} \lambda^{2} R^{\prime \prime \prime}(K ; \lambda+(1-\lambda) r) d \lambda .
\end{aligned}
$$


Then

$g_{K}(r)=\frac{\sqrt{r}\left(r^{2}+2 r+2+r^{-1}\right)}{(r+1)^{2}} R(K ; 1)+\frac{r \sqrt{r}}{(r+1)^{2}} J(K)+\frac{\sqrt{r} r(1-r)}{(r+1)^{2}} \int_{0}^{1} \lambda^{2} R^{\prime \prime \prime}(K ; \lambda+(1-\lambda) r) d \lambda$.

Integration by parts yields

$$
R^{\prime \prime \prime}(K ; r)=-r^{-4} \int w^{3} K^{\prime \prime}(w) K^{\prime}(w / r) d w .
$$

Then

$$
\int_{0}^{1} \lambda^{2} R^{\prime \prime \prime}(K ; \lambda+(1-\lambda) r) d \lambda \geq D(K) \int_{0}^{1} \frac{\lambda^{2}}{(\lambda+(1-\lambda) r)^{4}} d \lambda=\frac{D(K)}{3 r},
$$

which implies

$$
g_{K}(r) \geq\left[\left(r^{2.5}+2 r^{1.5}+2 r^{0.5}+r^{-0.5}\right) R(K ; 1)+r^{1.5} J(K)+\frac{1}{3}\left(r^{0.5}-r^{1.5}\right) D(K)\right] /(r+1)^{2} .
$$

Next we will find the points of the minimum of the lower bound for $g_{K}$. The denominator of the derivative of the lower bound is

$$
\left(1.5 r^{1.5}-1.5 r^{-0.5}-0.5 r^{-1.5}\right) R(K ; 1)+\left(1.5 r^{0.5}-0.5 r^{1.5}\right) J(K)+\left(\frac{1}{6} r^{-0.5}-r^{0.5}+\frac{1}{6} r^{1.5}\right) D(K)
$$

To find critical points we multiply by $r^{1.5}$ and obtain

$r^{3}\left(1.5 R(K ; 1)-0.5 J(K)+\frac{D(K)}{6}\right)+r^{2}(1.5 J(K)-D(K))+r\left(\frac{D(K)}{6}-1.5 R(K ; 1)\right)-0.5 R(K ; 1)=0$.

The cubic equation solver in MATLAB shows that this equation has only one real root when $R(K ; 1)>0, J(K)>0$. Thus one has to compare the values of the derivative at 0 , 0.3382 and 1 . They are $-0.5 R(K ; 1),-0.9493 R(K ; 1)+0.1522 J(K)-0.0516 D(K)$, and $-0.5 R(K ; 1)+J(K)-\frac{2 D(K)}{3}$, respectively. Only 3 cases are possible:

(a) If $-0.9493 R(K ; 1)+0.1522 J(K)-0.0516 D(K)>0$ and $-0.5 R(K ; 1)+J(K)-$ $\frac{2 D(K)}{3}>0$ then the point of minimum is 0.3382 . Note that the lower bound at 0.3382 equals $1.8665 R(K ; 1)+0.1098 J(K)+0.0716 D(K)$.

(b) If $-0.9493 R(K ; 1)+0.1522 J(K)-0.0516 D(K)<0$ and $-0.5 R(K ; 1)+J(K)-$ $\frac{2 D(K)}{3}>0$ then there exists the point of minimum $r_{0}$ in the interval $[0.3382,1]$. Then we substitute $D(K)>-18.3973 R(K ; 1)+2.9496 J(K)$ into the lower bound to obtain another lower bound

$$
R(K ; 1) \frac{r_{0}^{2.5}+8.1324 r_{0}^{1.5}-4.1324 r_{0}^{0.5}+r_{0}^{-0.5}}{\left(r_{0}+1\right)^{2}}+J(K) \frac{0.0168 r_{0}^{1.5}-0.9832 r_{0}^{0.5}}{\left(r_{0}+1\right)^{2}},
$$

which bounded from below by $0.5486 R(K ; 1)-0.3174 J(K)$, since $R(K ; 1)>0$ and $J(K)>$ 0 .

(c) If $-0.9493 R(K ; 1)+0.1522 J(K)-0.0516 D(K)<0$ and $-0.5 R(K ; 1)+J(K)-$ $\frac{2 D(K)}{3}<0$ then the point of minimum is 1 and the lower bound is equal to the upper bound.

The other case is impossible if $R(K ; 1)>0$ and $J(K)>0$, so the proof is complete. 


\section{References}

[1] Efromovich, S. Nonparametric curve estimation (1999) Springer-Verlag, New York.

[2] Hansen, B. E. (2005) Exact mean integrated squared error of higher order kernel estimators. Econometric Theory 21, 1031-1057.

[3] Jones, M.C. and Foster, P.J. (1993) Generalized jackknifing and higher order kernels. Journal of Nonparametric Statistics, 3, 81-94.

[4] Jones, M.C. and Signorini, D.F. (1997) A Comparison of Higher-Order Bias Kernel Density Estimators. Journal of Nonparametric Statistics 92, 1063-1073.

[5] Kim, C., Kim. S., Park, M., Lee, H. (2006) A bias reducing technique in kernel distribution function estimation. Computational Statistics, 21, 589-601.

[6] Mynbaev, K. and Martins-Filho, C. (2010), Bias reduction in kernel density estimation via Lipschitz condition. Journal of Nonparametric Statistics 22, 219-235.

[7] Sakhanenko, L. (2015) Rate Acceleration for Estimators of Integral Curves from Diffusion Tensor Imaging (DTI) Data. Statistics \& Probability Letters 107, 286-295.

[8] Schucany, W.R. and Sommers, J.P. (1977). Improvement of kernel type density estimators. J. Amer. Statist. Assoc. 72, 420-423.

[9] Scott, D. Multivariate density estimation: Theory, Practice, and Visualization (2015) Wiley Series in Probability and Statistics. 